\title{
Cardiometabolic comorbidities, readmission, and costs in schizophrenia and bipolar disorder: a real-world analysis
}

Christoph U. Correll ${ }^{1,2}$, Daisy S. Ng-Mak ${ }^{3 *}$, Dana Stafkey-Mailey ${ }^{4}$, Eileen Farrelly ${ }^{4}$, Krithika Rajagopalan ${ }^{3}$ and Antony Loebel ${ }^{5}$

\begin{abstract}
Background: Serious mental illnesses are associated with increased risk of cardiometabolic comorbidities. The objective of this study was to evaluate the prevalence of cardiometabolic comorbidity and its association with hospitalization outcomes and costs among inpatients with schizophrenia or bipolar disorder.

Methods: This retrospective database analysis reviewed patients with an inpatient diagnosis of schizophrenia or bipolar disorder from the Premier Perspective ${ }^{\circledR}$ Database (4/1/2010-6/30/2012). Patients were categorized into 4 cohorts based on the number of ICD-9-CM cardiometabolic comorbidities (i.e., 0, 1, 2, or 3+). Outcomes included length of stay, mortality during the index hospitalization, healthcare costs, and 30-day all-cause readmission rates.
\end{abstract}

Results: Of 57,506 patients with schizophrenia, $66.1 \%$ had at least one cardiometabolic comorbidity; $39.3 \%$ had two or more comorbidities. Of 124,803 patients with bipolar disorder, $60.5 \%$ had at least one cardiometabolic comorbidity; 33.4\% had two or more. Average length of stay was 8.5 (for patients with schizophrenia) and 5.2 (for patients with bipolar disorder) days. Each additional cardiometabolic comorbidity was associated with an increase in length of stay for patients with bipolar disorder $(p<.001)$ but not for patients with schizophrenia. Mortality rates during the index hospitalization were 1.2\% (schizophrenia) and .7\% (bipolar disorder). Each additional cardiometabolic comorbidity was associated with a significant increase in mortality for patients with bipolar disorder (OR 1.218, $p<.001$ ), and a numerical increase in mortality for patients with schizophrenia (OR 1.014, $p=.727)$. Patients with more cardiometabolic comorbidities were more likely to have a 30-day readmission (schizophrenia $=9-13 \%$; bipolar disorder $=7-12 \%$ ), and to incur higher costs (schizophrenia $=\$ 10,606-15,355$; bipolar disorder $=\$ 7126-13,523)$ (all $p<.01$ ).

Conclusions: Over $60 \%$ of inpatients with schizophrenia or bipolar disorder had cardiometabolic comorbidities. Greater cardiometabolic comorbidity burden was associated with an increased likelihood of readmission and higher costs among patients with schizophrenia or bipolar disorder, and an increase in length of stay and mortality for patients with bipolar disorder.

Keywords: Schizophrenia, Bipolar disorder, Cardiometabolic comorbidity, Hospitalization, Healthcare costs

\section{Background}

Severe and persistent mental illnesses that are often debilitating to patients, such as schizophrenia and bipolar disorder, are associated with increased physical

\footnotetext{
*Correspondence: daisy.ng-mak@sunovion.com

${ }^{3}$ Sunovion Pharmaceuticals Inc., 84 Waterford Dr., Marlborough, MA 01752, USA

Full list of author information is available at the end of the article
}

comorbidities and mortality [1-7]. Schizophrenia is characterized by psychosis, behavioral dysfunction, and cognitive impairment and has a prevalence of approximately $1 \%$ in the United States (US) [8]. Bipolar disorder is a mood disorder characterized by intermittent periods of mania and major depression that has an approximate lifetime prevalence of $4 \%$ among adults in the US [9]. The severe psychiatric symptoms and accompanying functional disability among patients that suffer from these 
debilitating disorders often result in high rates of unemployment [10], incarceration [11], and suicide [12].

Compounding the psychiatric disability in schizophrenia and bipolar disorder, a growing literature suggests that physical comorbidities in this population reduce life expectancy by as much as $10-25$ years and double the risk of premature mortality compared to the general population [7]. Patients with schizophrenia have 2.4 times the rate of metabolic syndrome and 2.0 times the rate of diabetes than the general population [1]. Similarly, patients with bipolar disorder have 2.0 times the rate of metabolic syndrome [2] and 1.7 times the rate of diabetes [3] than the general population. Furthermore, some evidence suggests that the prevalence of cardiometabolic risks is underestimated among patients with schizophrenia $[4,5]$ or bipolar disorder [9] due to under-diagnosis and under-treatment.

Complicating the inherent higher risk of cardiometabolic comorbidities in patients with schizophrenia or bipolar disorder, atypical antipsychotics, which are standard pharmacological treatment for schizophrenia and many patients with bipolar disorder, can exacerbate patients' risk of cardiometabolic disease [13]. For example, treatment with certain atypical antipsychotics is associated with an increasing risk of developing metabolic syndrome [14, 15], diabetes [16-19], and elevated low-density lipoprotein cholesterol levels [19-21].

Hospital readmissions within 30 days post-hospital discharges (i.e., 30-day readmissions) have become an important measure of health care quality due to the high 30-day readmission rates among US Medicare beneficiaries [22]. Evidence suggests that 30-day readmissions are a significant predictor of long-term mortality [23]. In order to reduce 30-day readmission rates, the Centers for Medicare and Medicaid Services implemented the Medicare Hospital Readmissions Reduction Program which, as a penalty, reduces Medicare payments to hospitals with excess 30 -day readmissions relative to the mean national readmission rates in conditions such as acute myocardial infarction and heart failure [24]. In a statistical brief summarizing the readmission trend in 2013, schizophrenia, mood disorder, and diabetes were among the top 20 conditions with the highest all-cause 30-day readmission rates [25].

While the prevalence, outcomes, and costs of cardiometabolic comorbidities in patients with schizophrenia and bipolar disorder have been examined in some outpatient studies [1-3], these variables remain relatively unexplored in hospitalized patients. In addition, although there is extensive literature regarding 30-day readmissions attributed to particular illnesses such as mental (e.g., schizophrenia) or cardiometabolic conditions (e.g., diabetes), the incremental impact of cardiometabolic conditions on readmission among patients with schizophrenia or bipolar disorder remains unknown. Therefore, the objectives of this study were to determine the prevalence of cardiometabolic comorbidities among inpatients with schizophrenia and bipolar disorder and to assess the role of incremental cardiometabolic comorbidity burden on length of stay, mortality, and healthcare costs during the initial admission. Following discharge, the study also examined the role of incremental cardiometabolic comorbidity burden on the 30-day readmission rate.

\section{Methods \\ Study design}

This retrospective observational study used administrative hospital data from the Premier Perspective Database $^{\circledR}$ (Premier, Inc., Charlotte, NC, USA) during the period from April 1, 2010 to June 30, 2012. The Premier database is the largest hospital administrative database in the US and provides detailed service information from over 700 geographically dispersed hospitals and over 50 million discharges since 2000. The database contained detailed service level information, diagnostic information, hospital characteristics, and patient demographic information. The database did not include any identifiable protected health information and, pursuant to the Health Insurance Portability and Accountability Act of 1996 [26], the study did not require institutional review board waiver or approval.

\section{Patient selection}

Patients with a primary, secondary, or admitting diagnosis of schizophrenia (International Classification of Diseases, 9th Revision, Clinical Modification [ICD-9-CM] code 295.xx) or bipolar disorder (ICD-9-CM codes 296.0, 296.1, 296.4-296.8, 301.11, or 301.13) coded during their hospitalization stays were identified between October 1, 2010 and May 31, 2012. The first such hospitalization record was designated as the patient's index hospitalization. Patients diagnosed with both schizophrenia and bipolar disorder, patients who were less than 18-year old, or patients who were transferred from another hospital or an unknown admission source were excluded from the analysis.

\section{Variable definitions}

Six cardiometabolic comorbidities were examined for both the patients with schizophrenia and bipolar disorder: cerebrovascular disease (ICD-9-CM 430-438.xx), coronary or ischemic heart disease (ICD-9-CM 410. $\mathrm{xx}$-411.xx, 413.xx-414.xx), diabetes mellitus (ICD-9-CM 250.xx), hyperglycemia (ICD-9-CM 790.2), hyperlipidemia (ICD-9-CM 272.x), and hypertension (ICD-9-CM 
401.x-405.x). Patients were categorized into 1 of 4 comorbidity cohorts based on the number of cardiometabolic comorbidity diagnoses recorded during their index hospitalization (i.e., 0,1 , 2, or $3+$ ). In addition to examining these specific cardiometabolic comorbidities, the Charlson Comorbidity Index (CCI) was coded based on an algorithm developed for administrative data [27] using comorbidities reported during the index hospitalization as well as all inpatient or outpatient hospitalizations in the Premier database that occurred in 6 months prior to the index admission.

\section{Outcome variables}

Outcome variables during the index hospitalization included length of stay, mortality, and costs. Following discharge from the index hospitalization, 30-day allcause readmission rates were also examined. Length of stay in days and mortality were obtained from the discharge record for the index hospitalization. The hospitals reported both the charges for each individual service based on their charge master and the costs the hospital reported incurring to deliver the services. This analysis focused on the costs to deliver services, which were split into pharmacy and medical costs with the medical costs representing all non-pharmacy costs. All costs were adjusted to 2014 US dollars using the medical care component of the Consumer Price Index from the US Bureau of Labor Statistics [28]. The 30-day all-cause readmission rates were defined as a subsequent readmission to the same hospital for any reason within 30 days of discharge.

\section{Statistical analyses}

Patient demographic and baseline characteristics were summarized using descriptive statistics. The relationship between cardiometabolic comorbidities and study outcomes were evaluated using multivariate statistical models controlling for the following baseline variables: age, gender, race, payer, CCI, hospital region (Midwest, Northeast, South, and West), hospital location (urban/ rural), hospital type (teaching/non-teaching), and hospital bed count. For the dichotomous outcome variables, mortality and 30-day readmission, logistic regression models were used. Length of stay was treated as count data and a negative binomial regression was used. Finally, for the highly skewed cost variables, total costs, pharmacy costs, and medical costs, generalized linear models (GLMs) with a gamma distribution and log-link function were used. The statistical models were fit separately for the schizophrenia and bipolar samples. Statistical analyses were conducted using SAS version 9.4 (SAS Institute, Cary, NC, USA). All analyses were two-sided with alpha of .05 .

\section{Results}

Patient selection

There were 118,065 patients with an inpatient hospitalization for schizophrenia. Of these, 51.3\% ( $n=60,559)$ were excluded for the following reasons: index hospitalization did not have the required 6-month prior observation or 1-month follow-up period; age younger than 18 years; transfer from another hospital or an unknown admission source; diagnoses of both schizophrenia and bipolar disorder. Among 229,974 patients with an inpatient hospitalization for bipolar disorder, $45.7 \%$ ( $n=105,171)$ were excluded after applying the exclusion criteria as above. The final study sample included 57,506 and 124,803 inpatients with a diagnosis of schizophrenia and bipolar disorder, respectively.

\section{Patient characteristics}

Patient characteristics are described in Table 1 . The average age of patients with schizophrenia and bipolar disorder was in the mid to late 40s. The most common cardiometabolic comorbidities were hypertension (52\% bipolar disorder; 57\% schizophrenia), hyperlipidemia (28\% bipolar disorder; $30 \%$ schizophrenia), and diabetes (22\% bipolar disorder; $28 \%$ schizophrenia), with each numerically higher for patients with schizophrenia than patients with bipolar disorder. Nearly two-thirds of patients with schizophrenia (66.1\%) and bipolar disorder (60.5\%) had at least one cardiometabolic comorbidity, and over one-third of patients with schizophrenia (39.3\%) and bipolar disorder (33.4\%) had 2 or more cardiometabolic comorbidities.

\section{Outcomes}

For the index hospitalization, the mean length of stay was 8.5 days for patients with schizophrenia and 5.2 days for patients with bipolar disorder. Multivariate analyses showed a negative association between cardiometabolic comorbidity burden with length of stay for schizophrenia $(p<.001)$, but a positive association for bipolar disorder $(p<.001)$ (see Fig. 1a).

The index hospitalization mortality rate was $1.2 \%$ for the patients with schizophrenia and .7\% for the patients with bipolar disorder. For those with schizophrenia, the risk of death during the index hospitalization was not significantly associated with each additional cardiometabolic comorbidity (odds ratio [OR] 1.014; 95\% confidence interval $[\mathrm{CI}] .937,1.098, p=.727)$. Prior to correcting for baseline differences, a Chi square test showed that patients with schizophrenia who had one or more cardiometabolic comorbidities had a higher risk of mortality compared to those with no comorbidities (1.7 vs. .3\%, $p<.001)$. For those with bipolar disorder, the risk of death during the index hospitalization increased by $21.8 \%$ (OR 
Table 1 Demographic, clinical, and hospital facility characteristics for patients with schizophrenia and bipolar disorder

\begin{tabular}{|c|c|c|}
\hline Characteristic & $\begin{array}{l}\text { Schizophrenia } \\
(N=57,506)\end{array}$ & $\begin{array}{l}\text { Bipolar disorder } \\
(N=124,803)\end{array}$ \\
\hline \multicolumn{3}{|l|}{ Demographics } \\
\hline Age, years (mean) & 49.8 & 45.4 \\
\hline Female (\%) & 43.0 & 63.0 \\
\hline \multicolumn{3}{|l|}{ Race (\%) } \\
\hline African American & 29.0 & 10.0 \\
\hline Caucasian & 51.0 & 73.0 \\
\hline Hispanic & 2.0 & 1.0 \\
\hline Other & 18.0 & 15.0 \\
\hline \multicolumn{3}{|l|}{ Region (\%) } \\
\hline Midwest & 24.0 & 24.0 \\
\hline Northeast & 23.0 & 20.0 \\
\hline South & 36.0 & 41.0 \\
\hline West & 17.0 & 15.0 \\
\hline \multicolumn{3}{|l|}{ Payer (\%) } \\
\hline Medicaid & 30.0 & 25.0 \\
\hline Medicare & 51.0 & 35.0 \\
\hline Commercial/private & 9.0 & 24.0 \\
\hline Self-pay & 6.0 & 9.0 \\
\hline Other & 5.0 & 6.0 \\
\hline \multicolumn{3}{|l|}{ Comorbidities } \\
\hline $\begin{array}{l}\text { Charlson comorbidity index } \\
\text { (mean) }\end{array}$ & 1.1 & 1.0 \\
\hline \multicolumn{3}{|l|}{$\begin{array}{l}\text { Specific cardiometabolic } \\
\text { comorbidities (\%) }\end{array}$} \\
\hline Diabetes & 28.0 & 22.0 \\
\hline Hyperglycemia & 2.0 & 2.0 \\
\hline Hypertension & 57.0 & 52.0 \\
\hline Hyperlipidemia & 30.0 & 28.0 \\
\hline Ischemic heart disease & 9.0 & 9.0 \\
\hline Cerebrovascular disease & 4.0 & 3.0 \\
\hline \multicolumn{3}{|c|}{ Number of cardiometabolic comorbidities (\%) } \\
\hline 0 & 33.9 & 39.5 \\
\hline 1 & 26.7 & 27.1 \\
\hline 2 & 19.9 & 16.6 \\
\hline $3+$ & 19.4 & 16.8 \\
\hline \multicolumn{3}{|l|}{ Hospital characteristics } \\
\hline Bed size (mean) & 436 & 409 \\
\hline Urban (\%) & 89.0 & 86.0 \\
\hline Teaching (\%) & 48.0 & 42.0 \\
\hline
\end{tabular}

1.218; 95\% CI 1.129, 1.314, $p<.001)$ with each additional cardiometabolic comorbidity (Fig. 1c). Prior to correcting for baseline differences, a Chi square test showed that patients with bipolar disorder who had one or more cardiometabolic comorbidities had a higher risk of mortality compared to those with no comorbidities (1.45 vs. .10\%, $p<.001)$.

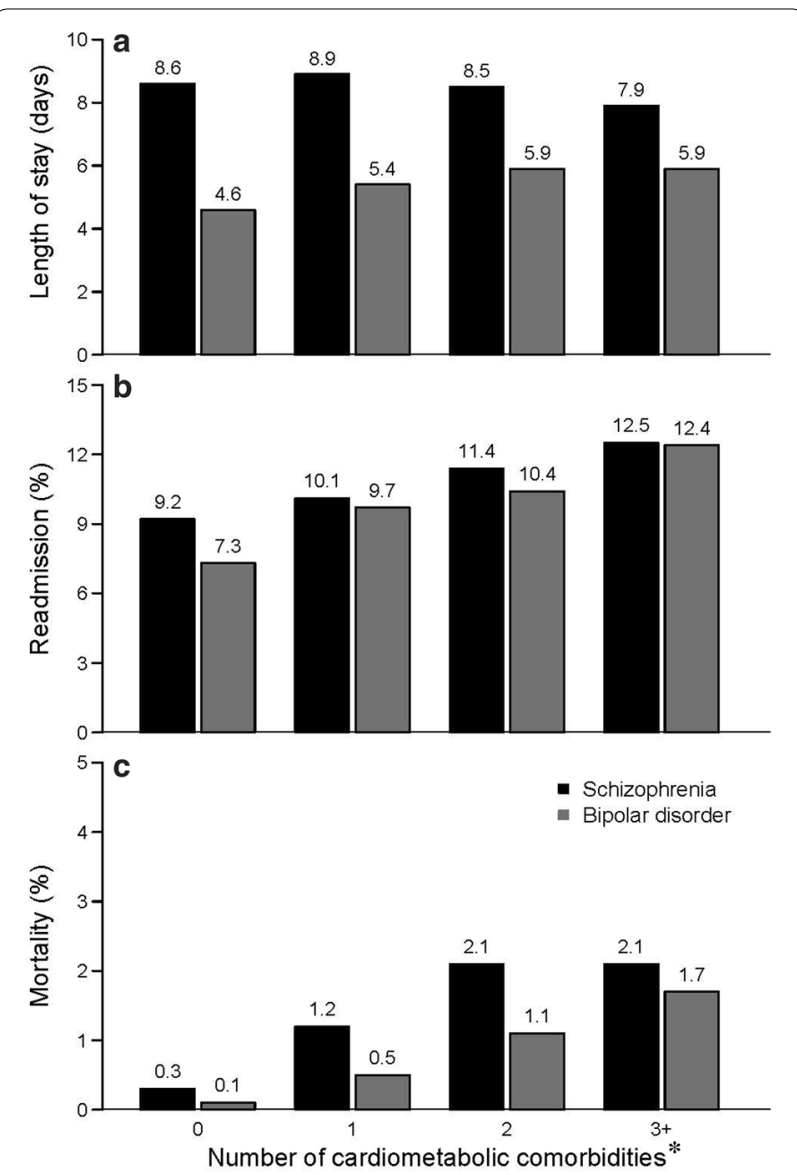

Fig. 1 Length of stay, 30-day all-cause readmission, and hospital mortality by number of cardiometabolic comorbidities. a The mean length of stay was 8.5 days for overall patients with schizophrenia and 5.2 days for patients with bipolar disorder. Negative binomial regressions showed a negative association between cardiometabolic comorbidity burden with length of stay for schizophrenia (-.015; $95 \% \mathrm{Cl}-.024,-.007, p<.001)$, but a positive association for bipolar disorder $(.029 ; 95 \% \mathrm{Cl} .024, .034, p<.001)$. b Overall, $11.8 \%$ of the patients with schizophrenia and $9.3 \%$ of the patients with bipolar disorder were readmitted for any reason within 30 days of discharge from the index hospitalization. For each additional cardiometabolic comorbidity, logistic regressions showed the odds of readmission increased by $3.1 \%$ (OR 1.031; $95 \% \mathrm{Cl} 1.001,1.061, p=.042)$ for schizophrenia and by $6.4 \%$ (OR 1.064; 95\% Cl 1.041, 1.087, $p<.001)$ for bipolar disorder. cThe index hospitalization mortality rate was $1.2 \%$ for overall patients with schizophrenia and .7\% for patients with bipolar disorder. In schizophrenia, cardiometabolic comorbidity was not significantly associated with mortality (OR 1.014; 95\% Cl .937, 1.098, $p=.727)$. A Chi square test showed that patients with schizophrenia who had one or more cardiometabolic comorbidities had a higher risk of mortality compared to those with no comorbidities (1.7 vs. .3\%, $p<.001)$. In bipolar disorder, each additional cardiometabolic comorbidity was associated with a $21.8 \%$ increase in mortality during the index hospitalization (OR 1.218; 95\% Cl 1.129, 1.314, p<.001). A Chi square test showed that patients with bipolar disorder who had one or more cardiometabolic comorbidities had a higher risk of mortality compared to those with no comorbidities ( 1.45 vs. .10\%, $p<.001$ ). * The following covariates were included in all regression analyses: age, gender, race, payer, CCl, hospital region, hospital location (urban/ rural), hospital type (teaching/non-teaching), and hospital bed count 
Hospitalization costs increased as the number of cardiometabolic comorbidities increased (see Fig. 2). For patients with schizophrenia, the mean total cost for the index hospitalization was $\$ 12,781$ per patient (medical and pharmacy costs of $\$ 11,771$ and $\$ 1010$ per patient, respectively). Medical costs increased by $6.8 \%$, pharmacy costs by $25.9 \%$, and total costs by $8.3 \%$ (all $p<.0001$ ) for each additional cardiometabolic comorbidity. For patients with bipolar disorder, the mean total cost for the index hospitalization was $\$ 9725$ per patient (medical and pharmacy costs of $\$ 8878$ and $\$ 847$ per patient, respectively). Medical costs increased by $12.3 \%$, pharmacy costs by $26.6 \%$, and total costs by $13.4 \%$ (all $p<.0001$ ) for each additional cardiometabolic comorbidity.

Within 30-days of discharge from the index hospitalization, $11.8 \%$ of the patients with schizophrenia and $9.3 \%$ of the patients with bipolar disorder were readmitted for any reason. Odds of readmission increased by $3.1 \%$ (OR 1.031; 95\% confidence interval [CI] 1.001, 1.061, $p=.042)$ for patients with schizophrenia and by $6.4 \%$ (OR $1.064 ; 95 \%$ CI $1.041,1.087, p<.001$ ) for patients with bipolar disorder (see Fig. 1b) with each additional cardiometabolic comorbidity.

\section{Discussion}

In this large, nationally representative administrative database study of hospitalized patients with schizophrenia and bipolar disorder, cardiometabolic comorbidities were common. Over $60 \%$ of patients had $\geq 1$ cardiometabolic comorbidity and over $30 \%$ had $\geq 2$ cardiometabolic comorbidities. Increasing cardiometabolic comorbidity burden was associated with a significantly higher mortality rate (for bipolar disorder), and longer hospital stays (for bipolar disorder). Patients with schizophrenia appeared to have almost double the rates of mortality in comparison to patients with bipolar disorder. The average total all-cause hospitalization cost was $\$ 12,781$ and $\$ 9725$ per patient for schizophrenia and bipolar disorder, respectively. Each incremental cardiometabolic comorbidity was associated with an 8.3 and $13.4 \%$ increase in total hospital cost for patients with schizophrenia and bipolar disorder, respectively. While 1 in 10 schizophrenia or bipolar disorder patients had an all-cause readmission within 30-days after index hospitalization, the odds of 30-day readmission increased with each incremental cardiometabolic comorbidity.

The reported frequencies of cardiometabolic comorbidities in this study are generally consistent with those previously reported in the literature [1, 2, 15, 29, 30]. However, this study identified a higher prevalence of diabetes in patients with schizophrenia and bipolar disorder (28 and $22 \%$, respectively) than previously reported (7-15\%) $[4,6,30-33]$. This discrepancy in the reported prevalence of diabetes may be due to the study population; previous studies have typically been drawn from

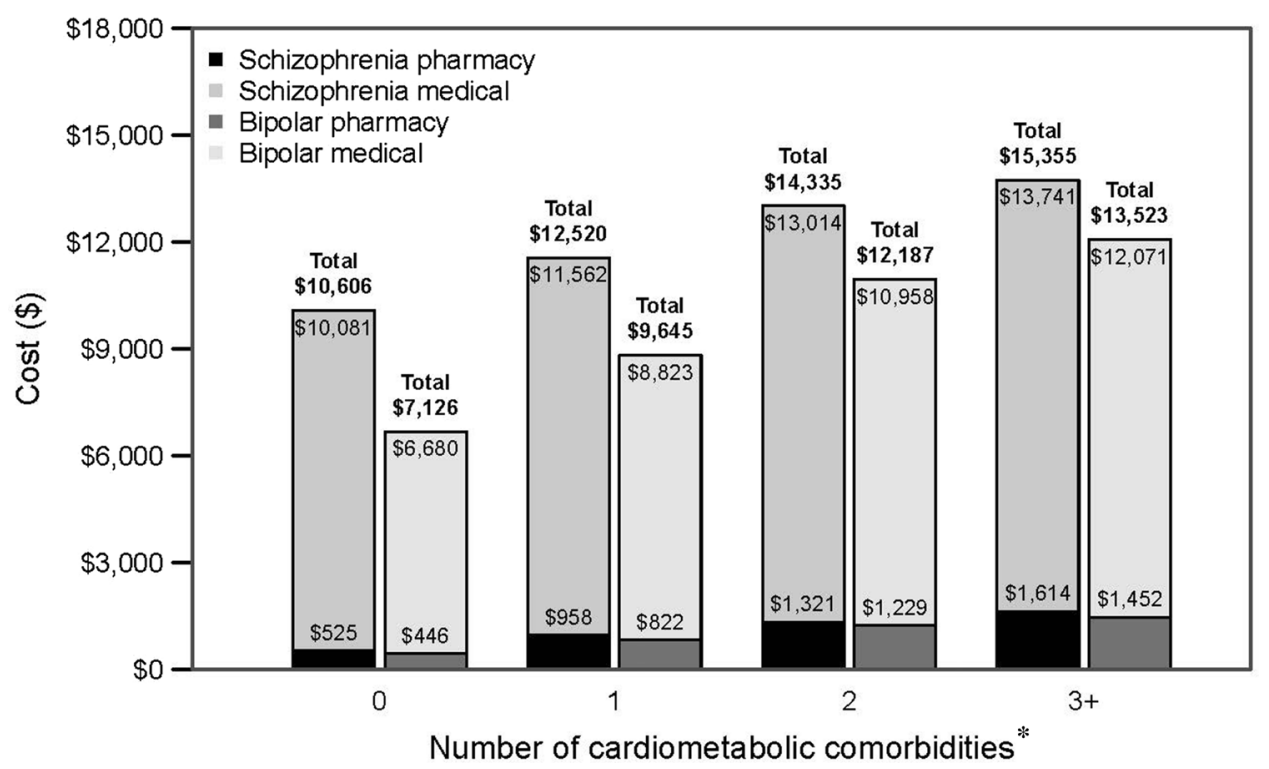

Fig. 2 Medical, pharmacy, and total costs by number of cardiometabolic comorbidities. Dollar figures reflect the costs to the hospital to deliver care in 2014 dollars. For both schizophrenia and bipolar disorder, increasing cardiometabolic comorbidity was associated with increased pharmacy, medical, and total index hospitalization costs (all $p<.001$ ). The following covariates were included in the gamma regression analyses with a log link: age, gender, race, payer, CCl, hospital region, hospital location (urban/rural), hospital type (teaching/non-teaching), and hospital bed count. * The data are presented by number of cardiometabolic comorbidities. Overall mean total cost for patients with schizophrenia was $\$ 12,781$ per patient (medical and pharmacy costs of $\$ 11,771$ and $\$ 1010$ per patient, respectively). Overall mean total cost for patients with bipolar disorder was $\$ 9725$ per patient (medical and pharmacy costs of $\$ 8878$ and $\$ 847$ per patient, respectively) 
broader health system populations [30], clinical trial participants [4], or primary care settings [6]. Among the general population, nearly $20 \%$ of hospital stays in the US are associated with diabetes [34]. The prevalence of diabetes found among inpatients with schizophrenia (28\%) or bipolar disorder $(22 \%)$ in this study is therefore plausible, given that they are already considered a high-risk population for diabetes [33].

To the best of our knowledge, this is the first study to evaluate the risk of 30-day readmission among hospitalized patients with schizophrenia or bipolar disorder and its association with incremental cardiometabolic comorbidity burden. Our study showed that incremental cardiometabolic comorbidity burden was associated with a 3.1 and $6.4 \%$ increased risk of early readmission in patients with schizophrenia and bipolar disorder, respectively. A recent chart review study of 945 patients hospitalized in a psychiatric care facility found that psychiatric readmission in the following year was independently predicted by higher body mass index (BMI) [35]. The authors hypothesized that inflammation, which has been associated with both higher BMI and obesity as well as psychiatric disorders [36] may represent the link between the greater BMI and need for readmission, but research examining the mechanisms of early readmissions and cardiometabolic comorbidities is needed.

For patients with bipolar disorder, additional cardiometabolic comorbidity burden was associated with an increase in the length of stay (4.6 days for no comorbidities to 5.9 days for $3+$ comorbidities). Surprisingly, increasing cardiometabolic comorbidity burden was associated with a small decrease in the length of stay among patients with schizophrenia (8.6 days for no comorbidities to 7.9 days for $3+$ comorbidities). While the reasons for these differences in length of stay are not known, it is possible that bipolar disorder patients may have been more likely to receive medical assessment and/or intervention for comorbid conditions than were patients with schizophrenia in this study; alternatively it is possible that patients with schizophrenia were more likely to have medical comorbidities that were well established and known to treatment staff compared to patients with bipolar disorder.

Prior research has clearly established a link between cardiometabolic conditions and mortality in the general population [37]. The lack of statistical significance between the odds of mortality and cardiometabolic comorbidity burden in schizophrenia after correcting for demographic and hospital characteristics was unexpected; however, a univariate analysis showed a significant association with comorbidity burden and mortality. Given the small sample sizes and the rarity of mortality incidence in this dataset, it is also plausible that the potential association between mortality and cardiovascular comorbidity is underestimated. Cardiovascular disease, along with cancer and suicide, has also been established as one of the leading causes of death for patients with schizophrenia [38]. Although information about the cause of death was unavailable, confounding of the results by suicide is likely small, as all the patients were hospitalized at community hospitals and not psychiatric hospitals, indicating severity of a medical, rather than psychiatric condition at the time of admission.

Previous studies have reported increased outpatient or total costs for psychiatric patients with cardiometabolic comorbidities [39-41]. This study is unique in that it demonstrated the possible relationship between each additional cardiometabolic comorbidity and incremental costs per admission for patients with schizophrenia or bipolar disorder.

The results of this study highlight the importance of identifying optimal treatment regimens for patients with serious mental illness. Efforts should be taken to adequately monitor for and reduce the rates of cardiometabolic comorbidities in this vulnerable patient population, and perhaps consider antipsychotic therapeutic options with a limited liability for such comorbidities [13]. From a holistic approach of treating patients with serious mental illness, clinicians should coordinate care and consider a patients' medical profile when prescribing medications. Coordinated care can also improve quality of care and patient satisfaction [42], which may have a positive effect on reducing healthcare costs through shorter hospital stays and/or reduced early readmissions. Furthermore, improved physical health may also positively impact psychiatric health outcomes [35]. In 2013, four new measures of the Healthcare Effectiveness Data and Information Set were added to assess quality of care for patients with serious mental illness; two of these four measures focus on diabetes monitoring and cardiovascular monitoring, which highlights the importance of monitoring cardiometabolic risks among this susceptible patient population [43]. Monitoring patients' cardiometabolic profiles, consideration of these risk factors when selecting antipsychotic drug therapies, and striving to coordinate care delivery for both mental and physical symptoms may maximize patients' outcomes.

\section{Limitations}

The data used in this study were collected for administrative reasons rather than for research purposes. The study design precludes any determination of causal relationship between cardiometabolic comorbidities and the outcomes. The analysis was restricted to variables present in this particular database, and other factors that were not available in the database may have confounded the observed relationships. Information about the prior treatments for the mental and physical morbidities, prior 
hospitalizations, and reasons for death was unavailable. In particular, data on atypical antipsychotic utilization prior to hospitalization were not available, therefore it was not possible to assess the relationship between specific antipsychotic medications and cardiometabolic comorbidities, which have been previously described to vary substantially $[2,13,20,33]$.

The burden of cardiometabolic comorbidities in this study was measured using diagnostic ICD-9-CM codes of 6 disease entities. Studies that have used the more robust National Cholesterol Education Program's Adult Treatment Panel III report (ATP III) or International Diabetes Federation (IDF) definitions [15] have reported metabolic syndrome prevalence of approximately $33 \%$ for schizophrenia [15] and 37\% for bipolar disorder [2]. The ICD-9-CM coding did not allow for the clear identification of bipolar II disorder (falls under "bipolar other"), precluding the assessment of differences in the number of cardiovascular comorbidities and outcomes between bipolar I and bipolar II subtypes. Moreover, there was no control group of inpatients without serious mental illness. The analysis of each patient was limited to a single index hospitalization and 30 days post-discharge, rather than attempting to determine hospital costs or outcomes over a longer duration of follow-up. Readmission rates in this study may be an underestimate, as these data only include admissions to the hospitals in the Premier network, and the likelihood of readmission to hospitals has been reported to be as high as $20 \%$ elsewhere [22].

\section{Conclusions}

In this large, retrospective, administrative database study, over $60 \%$ of patients with schizophrenia or bipolar disorder had at least one cardiometabolic comorbidity, and over $30 \%$ had two or more cardiometabolic comorbidities. For patients with schizophrenia, increasing cardiometabolic comorbidity burden had a significant impact on cost of index hospitalization, and 30-day readmission rates. For patients with bipolar disorder, increasing cardiometabolic comorbidity burden had a significant impact on length of stay, hospital mortality rates, cost of index hospitalization, and 30-day readmission rates. These results further underscore the need for improved detection and management of cardiometabolic risk factors in patients with schizophrenia or bipolar disorder across different clinical care settings. Further research is needed to better understand the longterm consequences of cardiometabolic comorbidity burden on patients with schizophrenia or bipolar disorder.

\section{Abbreviations}

ATP III: National Cholesterol Education Program's Adult Treatment Panel II; CCl: Charlson Comorbidity Index; GLMs: generalized linear models; ICD-9-CM: International Classification of Diseases, 9th Revision, Clinical Modification; IDF: International Diabetes Federation; US: United States.

\section{Authors' contributions}

Authors CUC, DSNM, DSM, EF, KR, and AL were involved in the study conception and design, drafting of the manuscript, data interpretation, and critical revision of the manuscript. Authors DSM and EF completed the data analysis in this study. All authors read and approved the final manuscript.

\section{Author details}

${ }^{1}$ Hofstra North Shore LIJ School of Medicine, Manhasset, NY, USA. ${ }^{2}$ The Zucker Hillside Hospital, Glen Oaks, NY, USA. ${ }^{3}$ Sunovion Pharmaceuticals Inc., 84 Waterford Dr., Marlborough, MA 01752, USA. ${ }^{4}$ Xcenda, Palm Harbor, FL, USA. ${ }^{5}$ Sunovion Pharmaceuticals Inc., Fort Lee, NJ, USA.

\section{Acknowledgements}

The authors acknowledge the contributions of Dr. Mariam Hassan and Dr. Chien-Chia Chuang who contributed to the design of this study and provided editorial support, respectively. Charles Meyer, who received support from Xcenda, and Dr. Michael Stensland of Agile Outcomes Research, Inc. both provided writing support.

\section{Competing interests}

Authors DSNM, KR, and AL are all employees of Sunovion Pharmaceuticals Inc. Other authors (DSM and EF) are employees of Xcenda LLC, which was compensated by Sunovion Pharmaceuticals Inc. All competing interests have been disclosed.

\section{Availability of data and materials}

The secondary data that support the findings of this study were obtained under license for the current study from Premier Perspective Database ${ }^{\circledR}$ (Premier, Inc., Charlotte, NC, USA), the largest hospital-based, service-level database in the US. The Premier database contained detailed service information from over 500 hospitals for more than 50 million inpatient discharges since the year 2000. Data supporting this study are available from the authors upon reasonable request and with permission of Premier Perspective Database ${ }^{\circledR}$ (Premier, Inc., Charlotte, NC, USA).

\section{Funding}

This research was funded by Sunovion Pharmaceuticals Inc., Marlborough, MA, USA. The authors who were employees of Sunovion Pharmaceuticals Inc. were involved in the final decision to publish study results and the sponsor reviewed the manuscript prior to submission, but publication of study results was not contingent on the sponsor's approval or censorship of the manuscript.

Received: 26 October 2016 Accepted: 1 February 2017

Published online: 10 February 2017

References

1. Vancampfort $D$, Wampers M, Mitchell AJ, et al. A meta-analysis of cardiometabolic abnormalities in drug naïve, first-episode and multi-episode patients with schizophrenia versus general population controls. World Psychiatry. 2013;12(3):240-50

2. Vancampfort D, Vansteelandt K, Correll CU, et al. Metabolic syndrome and metabolic abnormalities in bipolar disorder: a meta-analysis of prevalence rates and moderators. Am J Psychiatry. 2013;170(3):265-74.

3. Crump C, Sundquist K, Winkleby MA, Sundquist J. Comorbidities and mortality in bipolar disorder: a Swedish national cohort study. JAMA Psychiatry. 2013:70(9):931-9.

4. Nasrallah HA, Meyer JM, Goff DC, et al. Low rates of treatment for hypertension, dyslipidemia and diabetes in schizophrenia: data from the CATIE schizophrenia trial sample at baseline. Schizophr Res. 2006:86(1-3):15-22.

5. Correll CU, Robinson DG, Schooler NR, et al. Cardiometabolic risk in first episode schizophrenia-spectrum disorder patients: baseline results from the RAISE-ETP study. JAMA Psychiatry. 2014;71(12):1350-63.

6. Smith DJ, Martin D, McLean G, Langan J, Guthrie B, Mercer SW. Multimorbidity in bipolar disorder and undertreatment of cardiovascular disease: a cross sectional study. BMC Med. 2013:11:263. 
7. Nordentoft M, Wahlbeck K, Hällgren J, et al. Excess mortality, causes of death and life expectancy in 270,770 patients with recent onset of mental disorders in Denmark, Finland and Sweden. PLoS ONE. 2013;8(1):e55176.

8. Narrow WE, Rae DS, Robins LN, Regier DA. Revised prevalence estimates of mental disorders in the United States: using a clinical significance criterion to reconcile 2 surveys' estimates. Arch Gen Psychiatry. 2002;59(2):115-23.

9. Merikangas KR, Akiskal HS, Angst J, et al. Lifetime and 12-month prevalence of bipolar spectrum disorder in the National Comorbidity Survey replication. Arch Gen Psychiatry. 2007;64(5):543-52.

10. Jääskeläinen $\mathrm{E}$, Juola $\mathrm{P}$, Hirvonen $\mathrm{N}$, et al. A systematic review and meta-analysis of recovery in schizophrenia. Schizophr Bull. 2013;39(6):1296-306.

11. Thornicroft G, Tansella M, Becker T, et al. The personal impact of schizophrenia in Europe. Schizophr Res. 2004;69(2-3):125-32.

12. Olfson M, Gerhard T, Huang C, Crystal S, Stroup TS. Premature mortality among adults with schizophrenia in the United States. JAMA Psychiatry. 2015;72(12):1172-81.

13. De Hert M, Detraux J, van Winkel R, Yu W, Correll CU. Metabolic and cardiovascular adverse effects associated with antipsychotic drugs. Nat Rev Endocrinol. 2011;8(2):114-26.

14. Meyer JM, Davis VG, Goff DC, et al. Change in metabolic syndrome parameters with antipsychotic treatment in the CATIE Schizophrenia Trial: prospective data from phase 1. Schizophr Res. 2008;101(1-3):273-86.

15. Mitchell AJ, Vancampfort D, Sweers K, van Winkel R, Yu W, De Hert M. Prevalence of metabolic syndrome and metabolic abnormalities in schizophrenia and related disorders - a systematic review and metaanalysis. Schizophr Bull. 2013;39(2):306-18.

16. Smith M, Hopkins D, Peveler RC, Holt RI, Woodward M, Ismail K. First-v. second-generation antipsychotics and risk for diabetes in schizophrenia: systematic review and meta-analysis. Br J Psychiatry. 2008;192(6):406-11.

17. Nielsen J, Skadhede S, Correll CU. Antipsychotics associated with the development of type 2 diabetes in antipsychotic-naïve schizophrenia patients. Neuropsychopharmacology. 2010;35(9):1997-2004.

18. Ulcickas Yood M, Delorenze GN, Quesenberry CP Jr, et al. Association between second-generation antipsychotics and newly diagnosed treated diabetes mellitus: does the effect differ by dose? BMC Psychiatry. 2011;1:197.

19. American Diabetes Association, American Psychiatric Association, American Association of Clinical Endocrinologists, North American Association for the Study of obesity. Consensus development conference on antipsychotic drugs and obesity and diabetes. Diabetes Care. 2004;27(2):596-601

20. Correll CU, Lencz T, Malhotra AK. Antipsychotic drugs and obesity. Trends Mol Med. 2011;17(2):97-107.

21. Rummel-Kluge C, Komossa K, Schwarz S, et al. Head-to-head comparisons of metabolic side effects of second generation antipsychotics in the treatment of schizophrenia: a systematic review and meta-analysis. Schizophr Res. 2010;123(2-3):225-33.

22. Jencks SF, Williams MV, Coleman EA. Rehospitalizations among patients in the Medicare fee-for-service program. N Engl J Med. 2009;360(14):1418-28.

23. Yian E, Zhou H, Schreiber A, et al. Early hospital readmission and mortality risk after surgical treatment of proximal humerus fractures in a community-based health care organization. Perm J. 2016;20(1):47-52.

24. Centers for Medicare \& Medicaid Services: Readmissions Reduction Program. https://www.cms.gov/medicare/medicare-fee-for-servicepayment/acuteinpatientpps/readmissions-reduction-program.html. Accessed 3 Aug 2016.

25. Fingar $\mathrm{K}$, Washington R. Trends in hospital readmissions for four highvolume conditions, 2009-2013: Statistical Brief \#196. Rockville: Agency for Healthcare Research and Quality. 2015. https://www.hcup-us.ahrq.gov/ reports/statbriefs/sb 196-Readmissions-Trends-High-Volume-Conditions. jsp. Accessed 3 Aug 2016.

26. United States Congress. Health Insurance Portability and Accountability Act of 1996. 1996. http://www.gpo.gov/fdsys/pkg/PLAW-104publ191/ html/PLAW-104publ191.htm. Accessed 3 Aug 2016.

27. Deyo RA, Cherkin DC, Ciol MA. Adapting a clinical comorbidity index for use with ICD-9-CM administrative databases. J Clin Epidemiol. 1992;45(6):613-9.
28. U.S. Bureau of Labor Statistics. Measuring price change for medical care in the CPI. 2009. http://www.bls.gov/cpi/cpifact4.htm. Accessed 3 Aug 2016.

29. Kemp DE, Sylvia LG, Calabrese JR, et al, LiTMUS Study Group. General medical burden in bipolar disorder: findings from the LiTMUS comparative effectiveness trial. Acta Psychiatr Scand. 2014;129:24-34.

30. Bresee LC, Majumdar SR, Patten SB, Johnson JA. Prevalence of cardiovascular risk factors and disease in people with schizophrenia: a populationbased study. Schizophr Res. 2010;117(1):75-82.

31. Cohen D, Stolk RP, Grobbee DE, Gispen-de Wied CC. Hyperglycemia and diabetes in patients with schizophrenia or schizoaffective disorders. Diabetes Care. 2006;29(4):786-91.

32. van Winkel $R$, De Hert $M$, Van Eyck D, et al. Prevalence of diabetes and the metabolic syndrome in a sample of patients with bipolar disorder. Bipolar Disord. 2008;10(2):342-8.

33. Vancampfort D, Correll CU, Galling B, et al. Diabetes mellitus in people with schizophrenia, bipolar disorder and major depressive disorder: a systematic review and large scale meta-analysis. World Psychiatry. 2016;15(2):166-74.

34. Fraze TK, Jiang HJ, Burgess J. Hospital stays for patients with diabetes, 2008. HCUP Statistical Brief \#93. Rockville: Agency for Healthcare Research and Quality. 2010. http://www.hcup-us.ahrq.gov/reports/statbriefs/sb93. pdf. Accessed 3 Aug 2016.

35. Manu P, Khan S, Radhakrishan R, Russ MJ, Kane JM, Correll CU. Body mass index identified as an independent predictor of psychiatric readmission. $J$ Clin Psychiatry. 2014;75(6):e573-7.

36. Manu P, Correll CU, Wampers M, et al. Markers of inflammation in schizophrenia: association vs. causation. World Psychiatry. 2014;13(2):189-92.

37. Casey DE, Haupt DW, Newcomer JW, Henderson DC, Sernyak MJ, Davidson M, Lindenmayer J-P, Manoukian SV, Banerji MA, Lebovitz HE, Hennekens $\mathrm{CH}$. Antipsychotic-induced weight gain and metabolic abnormalities: implications for increased mortality in patients with schizophrenia. J Clin Psychiatry. 2004;65(S7):4-18.

38. Bushe CJ, Taylor M, Haukka J. Mortality in schizophrenia: a measurable clinical endpoint. J. Psychopharmacol. Oxf. Engl. 2010;24:17-25.

39. Chwastiak LA, Rosenheck RA, McEvoy JP, et al. The impact of obesity on the costs among persons with schizophrenia. Gen Hosp Psychiatry. 2009:31(1):1-7.

40. Jerrell JM, McIntyre RS, Tripathi A. Incidence and costs of cardiometabolic conditions in patients with schizophrenia treated with antipsychotic medications. Clin Schizophr Relat Psychoses. 2010;4(3):161-8.

41. Guo JJ, Keck PE, Li H, Patel NC. Treatment costs related to bipolar disorder and comorbid conditions among Medicaid patients with bipolar disorder. Psychiatr Serv. 2007:58(8):1073-8.

42. Horvitz-Lennon M, Kilbourne AM, Pincus HA. From silos to bridges: meeting the general health care needs of adults with severe mental illnesses. Health Aff (Millwood). 2006;25(3):659-69.

43. National Committee for Quality Assurance. HEDIS 2013: Technical Specifications for Health Plans. 2012. http://www.ncqa.org.

\section{Submit your next manuscript to BioMed Central and we will help you at every step:}

- We accept pre-submission inquiries

- Our selector tool helps you to find the most relevant journal

- We provide round the clock customer support

- Convenient online submission

- Thorough peer review

- Inclusion in PubMed and all major indexing services

- Maximum visibility for your research

Submit your manuscript at www.biomedcentral.com/submit 